\title{
Targeting Viral mRNA Translation Control as a New Concept for Anti-Virus Therapeutic Strategies
}

\author{
Chrysoula Daskalogianni ${ }^{1,2}$, Alice Zeng1, Maria Tovar-Fernandez ${ }^{1,2}$, Katerina Thermou ${ }^{1,2}$, Ewa Sroka ${ }^{1,2}$, Sara Mikac $^{2}$, Nassima $^{1}$ \\ Oumata $^{4}$, Hervé Galons ${ }^{5}$ and Robin Fahraeus ${ }^{1,2,3,6,7 *}$ \\ France \\ ${ }^{2}$ ICCVS, University of Gdańsk, Science, ul. Wita Stwosza 63, 80-308 Gdańsk, Poland \\ ${ }^{3}$ Inserm UMRS1131, Institut de Génétique Moléculaire, Université Paris 7, Hôpital St. Louis, F-75010 Paris, France \\ ${ }^{4}$ Université Bretagne Sud, 10 Rue Jean Zay, CS 55564, 56100 Lorient \\ ${ }^{5}$ Université de Paris, 4, avenue de l'Observatoire, 75006 Paris, France \\ ${ }^{6}$ RECAMO, Masaryk Memorial Cancer Institute, Zluty kopec 7, 65653 Brno, Czech Republic \\ ${ }^{7}$ Department of Medical Biosciences, Building 6M, Umeå University, 90185 Umeå, Sweden
}

${ }^{1}$ Université Paris-Saclay, Institut Gustave Roussy, Inserm UMR1015, Immunologie des tumeurs et Immunothérapie contre le cancer, 94805 Villejuif,

${ }^{\star}$ Corresponding author: Robin Fahraeus, Université Paris-Saclay, Institut Gustave Roussy, Inserm UMR1015, Immunologie des tumeurs et Immunothérapie contre le cancer, 94805 Villejuif, France; E-mail: robin.fahraeus@inserm.fr

Received: July 26, 2021; Accepted: August 06, 2021; Published: August 16, 2021

It was long thought that the ribosome and mRNA translation were not suitable targets for treatments against specific diseases. This was partly due to the fact that antibiotics targeting the protein synthesis machinery are not mRNA specific and it was also thought that regulation of gene expression was mainly regulated on the level of transcription and that mRNAs were, indeed, just messengers. These views have now changed and we now know that mRNA translation is a highly sophisticated and regulated process and that drugs can target the synthesis of specific proteins [1-3]. Biotech companies and also larger pharmaceutical companies are now investing more in trying to control gene expression on a post transcriptional level. The signalling pathway with, for example, mTOR has been a target for translation control but the selective regulation of individual messenger RNAs is less developed.

Viruses have evolved highly specialised factors to specifically interfere with their host cell environment. The specificity of these interactions is well illustrated by the potential fatal effects that can occur when a viruses jump species. The virus-host interaction is also well illustrated by the specificity by which latent viruses chose not only the host but also the tissue type. For example, the two gamma herpes viruses Epstein-Barr (EBV) and Kaposi sarcoma virus (KSHV), also referred to as human herpes virus 8 (HHV-8) are quite similar but have slightly different host cell preferences and cause different diseases [4-6]. Both viruses establish asymptomatic latency in the B cell population and in the case of EBV this is, from the virus point of view, a complete success story with approximately $90 \%$ of the population worldwide infected, making it practically a part of our genome. EBV infection from adolescence an onwards can cause mononucleosis and in the case of the endemic form of Burkitt's lymphoma the EBV is present in $100 \%$ of the cases. EBV infection of epithelial cells of the nasopharynx can also cause cancers and KSHV-infected endothelial cells are linked to Kaposi sarcoma in immune suppressed patients.
Latent viruses, in particular, have evolved ingenious mechanisms to evade the immune system. Normally, antigenic peptide substrates are processed by the cells and presented on major histocompatibility class I (MHC-I) molecules. These are scanned by cytotoxic T cells and if recognised, the antigen peptide-producing cell is eliminated [7]. More recent works have shown that what the immune system actually is focused on in terms of detecting virus infection is the presence of viral mRNAs and not the corresponding full length proteins [8]. Furthermore, it is not even the full length proteins encoded by these messages that are used by the immune system but smaller open reading frames translated by a non-canonical mRNA translation event. The benefit of this for the host is that the first peptides derived from an mRNA are destined for the class I pathway, giving the viruses less opportunities to produce factors that interfere with the antigen presentation pathway. Hence, mRNA translation is the key for nonself recognition. Viruses, such as EBV and KSHV, have evolved clever mechanisms to adapt and circumvent in the everlasting cat and mouse game between parasites and the immune system, so that they can express the essential latent gene products EBNA1 and LANA1, respectively, for viral genome maintenance without attracting the attention of the immune system $[9,10]$. This immune evasion is achieved by cis-acting mechanisms whereby the viral mRNAs suppress their own translation to minimize the production of antigenic peptide substrates and, at the same time, the encoded proteins have a low turnover rate in order to support viral functions. The molecular mechanisms of this cis-acting suppression of EBNA1 and LANA1 synthesis are based around RNA structures within the coding sequences [11]. Both RNAs contain G quadruple (G4) structures that control RNA processing as well as how the RNAs are translated. Nucleolin is a RNA-binding protein that plays a key role in suppressing synthesis of these RNAs and reducing nucleolin levels in the cells increase the expression of EBNA1 and stimulates the amount of antigenic peptide substrates produced for the 


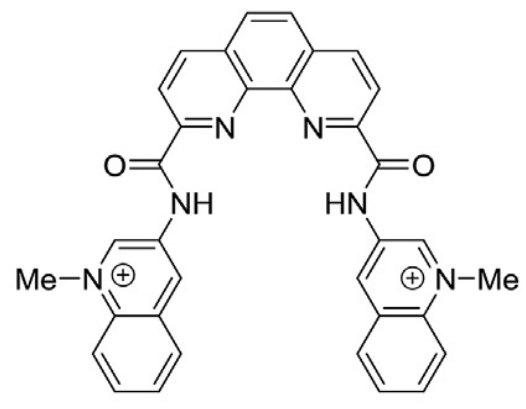

PhenDC3

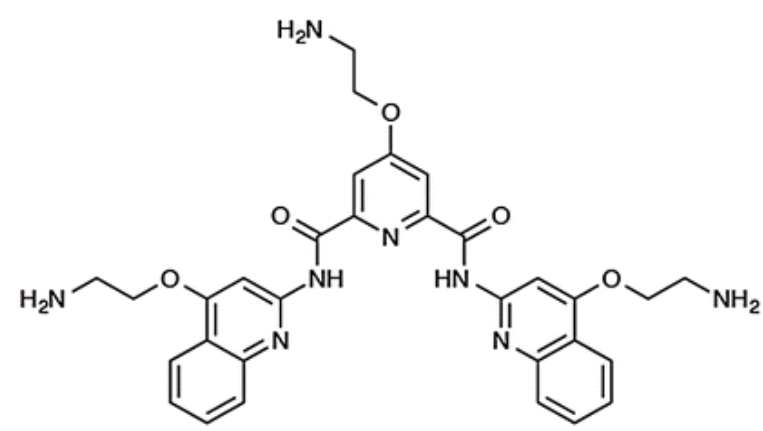

Figure : The G4 ligands PhenDC3 (left) displaces nucleolin from the EBNA1 mRNA, promotes nuclear export and stimulates EBNA1 mRNA translation and the synthesis of EBNA1-derived antigenic peptides. The G4 ligand Pyridostatin (PDS) (right) has no effect. If the G4 structure of the EBNA1 message is removed, PhenDC3 does not affect EBNA1 synthesis.

MHC-I pathway. In line with this, treating cells with small chemical G4 ligands (PhenDC3 or PhenD2) that displace nucleolin stimulate EBNA1 translation. Deletion of the G4-encoding sequence in the EBNA1 message diminishes the effect of the drug, illustrating that these compounds do not have a general effect on protein expression. Interestingly, there are several G4 ligands developed and the PDS, or Pyridostatin, has different chemical structure (see below). PDS does not displace nucleolin and has no effect on the translation of the EBNA1 mRNA or on the production of antigenic peptides for the class I pathway [12]. There is no apparent toxicity of PhenDC3 and $\mathrm{PhD} 2$ compounds in animals and they have no general effect on protein synthesis, suggesting that this could be a therapeutic approach to increase the immunogenicity of cancer cells harbouring EBV or KHSV. Even if these compounds are less suitable for drug development they have served the proof of concept, demonstrating that translation of specific viral mRNAs can be a drug target with the aim to overcome viral immune evasive mechanisms.

If this strategy of immune evasion adapted by EBV and KSHV is successful, the question is if other latent viruses are using similar strategies and mechanisms to target mRNA translation in cis. Human cytomegalovirus (CMV) is another herpesvirus linked to various forms of human disease and, like EBV, it causes problems in patients undergoing immunosuppression, for example, in connection with organ transplantation. However, CMV differs from EBV and KSHV and it latency program and gene expression pattern during latency is complicated. A vaccine has been developed against the more transforming variants of human papilloma virus (HPV) to prevent against HPV-derived cancers. Vaccines against the HPV-encoded E6/ E7 are also being developed for cancer treatments, illustrating how important the immune system is to prevent and suppress these types of cancers. It is thus conceivable that HPV also employs immune suppressing strategies to reduce its visibility [13]. There are no obvious similarities between the sequences of E6/E7 on the one hand and EBNA1 and LANA1 on the other, suggesting that even though the concept used by the different viruses might be similar, the mechanisms might differ. These are just a few examples of human virus for which it would be interesting to know more about cis-acting mechanisms of immune evasion and potential opportunities for therapeutic interference but it is also worthwhile keeping in mind that viruses are also a major health problem in domesticated farm animals and pets.

\section{Acknowledgments}

This work was partially supported by Inserm, European Regional Development Fund (ENOCH, CZ.02.1.01/0.0/0.0/16_019/0000868), MH CZ - DRO (MMCI, 00209805), Cancerforskningsfonden Norr, Cancerfonden (160598), Vetenskapsradet and by the International Centre for Cancer Vaccine Science within the International Research Agendas program of the Foundation for Polish Science co-financed by the European Union under the European Regional Development Fund.

Keywords: MHC class I pathway, mRNA translation, Therapeutics, Viral immune evasion

\section{References}

1. Yu AM, Choi YH, Tu MJ (2020) RNA Drugs and RNA Targets for Small Molecules: Principles, Progress, and Challenges. Pharmacological reviews 72: 862-898.

2. Pal I, Safari M, Jovanovic M, Bates SE, Deng C (2019) Targeting Translation of mRNA as a Therapeutic Strategy in Cancer. Current hematologic malignancy reports 14: 219227. [crossref]

3. Malina A, Mills JR, Pelletier J (2012) Emerging therapeutics targeting mRNA translation. Cold Spring Harbor perspectives in biology 4: a012377. [crossref]

4. Daskalogianni C, Pyndiah S, Apcher S, Mazars A, Manoury B, et al. (2015) EpsteinBarr virus-encoded EBNA1 and ZEBRA: targets for therapeutic strategies against EBV-carrying cancers. The Journal of pathology 235: 334-341. [crossref]

5. Young LS, Yap LF, Murray PG (2016) Epstein-Barr virus: more than 50 years old and still providing surprises. Nature reviews Cancer 16: 789-802. [crossref]

6. Cesarman E, Damania B, Krown SE, Martin J, Bower M, et al. (2019) Kaposi sarcoma Nature reviews. Disease primers 5: 9. [crossref]

7. Shastri N, Schwab S, Serwold T (2002) Producing nature's gene-chips: the generation of peptides for display by MHC class I molecules. Annu Rev Immunol 20: 463-493. [crossref]

8. Yewdell JW, Dersh D, Fahraeus R (2019) Peptide Channeling: The Key to MHC Class I Immunosurveillance? Trends in cell biology 29: 929-939.

9. Kwun HJ, da Silva SR, Shah IM, Blake N, Moore PS, et al. (2007) Kaposi's sarcomaassociated herpesvirus latency-associated nuclear antigen 1 mimics Epstein-Barr virus EBNA1 immune evasion through central repeat domain effects on protein processing. J Virol 81: 8225-8235. [crossref]

10. Yin Y, Manoury B, Fahraeus R (2003) Self-inhibition of synthesis and antigen presentation by Epstein-Barr virus-encoded EBNA1. Science 301: 1371-1374. [crossref]

11. Lista MJ, Martins RP, Billant O, Contesse MA, Findakly S, et al. (2017) Nucleolin directly mediates Epstein-Barr virus immune evasion through binding to G-quadruplexes of EBNA1 mRNA. Nature communications 8: 16043. [crossref] 
Robin Fahraeus (2021) Targeting Viral mRNA Translation Control as a New Concept for Anti-Virus Therapeutic Strategies

12. Reznichenko O, Quillevere A, Martins RP, Loaec N, Kang H, et al. (2019) Novel cationic bis(acylhydrazones) as modulators of Epstein-Barr virus immune evasion acting through disruption of interaction between nucleolin and G-quadruplexes of EBNA1 mRNA. European journal of medicinal chemistry 178: 13-29. [crossref]
13. Roden RB, Ling M, Wu TC (2004) Vaccination to prevent and treat cervical cancer. Human pathology 35: 971-982. [crossref]

\section{Citation:}

Daskalogianni C, Zeng A, Tovar-Fernandez M, Thermou K, Sroka E, et al. (2021) Targeting Viral mRNA Translation Control as a New Concept for Anti-Virus Therapeutic Strategies. Infect Dis Ther Volume 2(2): 1-3. 